\title{
Étude numérique d'un écoulement externe faiblement ionisé en non-équilibre chimique, vibrationnel et électronique
}

\author{
${\text { Ghislain } \text { Thhuen }^{1, a} \text {, Yves Burtschell }}^{2}$ et David E. Zeitoun ${ }^{2}$ \\ 1 Institut universitaire de technologie Fotso Victor, Université de Dschang, BP 134, Bandjoun, Cameroun \\ 2 École polytechnique universitaire de Marseille, DME, 5 rue Enrico Fermi, Technopole de Chateau Gombert, \\ 13453 Marseille Cedex 13, France
}

Reçu le 14 octobre 2004, accepté le 21 juin 2005

\begin{abstract}
Résumé - Cette étude traite de la simulation numérique des écoulements à haute enthalpie en nonéquilibre thermochimique. La résolution des équations 2D-axisymétrique de Navier-Stokes permet de prévoir numériquement la structure d'un écoulement réactif, partiellement ionisé. La méthode numérique repose sur une formulation de type volume fini couplée à l'approche MUSCL-TVD et est associée à un solveur de Riemann. L'intérêt est porté sur la contribution des différents phénomènes de non-équilibre sur le flux de chaleur et les paramètres thermodynamiques. L'écoulement est externe et s'effectue autour du nez d'un sphère-cylindre se déplaçant avec des nombres de Mach compris entre 15 et 18. Les résultats obtenus sont en bon accord avec les valeurs prédites par d'autres simulations et les données expérimentales.
\end{abstract}

Mots clés : Écoulement réactif / hypersonique / non-équilibre thermochimique / gaz ionisé / vibration / électronique

\begin{abstract}
Numerical study of a weakly ionized external flow in chemical, vibrational and electronic nonequilibrium. This study treats the numerical simulation of high enthalpy nonequilibrium hypersonic flow. The computation of a 2D axisymmetric partially ionized flow around blunt body have been investigated, using time dependent Navier-Stokes equations solved numerically. The equations were discretized by using a multiblock MUSCL-TVD finite volume scheme, coupling with the Riemann's solvers flux splitting approach. The upstream test cases Mach number varied from 15 to 18. Numerical results of thermodynamic parameters, and predicted heat flux around considered configurations have been well compared with previous simulations and experimental data.
\end{abstract}

Key words: Reactive flow / hypersonic / thermochemical nonequilibrium / ionized gas / vibration / electronic

\section{Introduction}

L'un des problèmes majeurs dans la réalisation d'engins de rentrée atmosphérique est la protection aérothermique afin de maintenir supportable pour l'homme, la température à l'intérieur de l'habitacle. Des travaux très précis dans ce domaine sont motivés par la volonté des constructeurs, d'affiner la connaissance et la prévision des flux de chaleur maximaux lors des phases de rentrée afin de réduire les marges de sécurité et optimiser la masse de la protection thermique au profit de celle de la charge utile. La désintégration de la navette Columbia entraînant la mort de 7 astronautes le samedi

\footnotetext{
a Auteur correspondant : tchuengse@yahoo.com
}

$1^{\text {er }}$ février 2003 dans le ciel du Texas aux USA illustre encore à nos jours de manière tragique la complexité des travaux à réaliser.

Lors des phases de rentrée, l'engin spatial vole à très grande vitesse, à très haute altitude et traverse plusieurs couches atmosphériques plus ou moins denses. Un choc détaché se forme à l'avant du véhicule au travers duquel l'écoulement ralenti et une partie importante de l'énergie cinétique est transférée au gaz sous forme d'énergie interne, ce qui se traduit par une élévation brutale de température. Par conséquent, la couche de choc est le siège d'intenses processus physico-chimiques tels que l'excitation vibrationnelle des molécules suivie de leur dissociation, l'excitation électronique des espèces chimiques, l'ionisation pour des températures très élevées 


\section{Nomenclature}

\begin{tabular}{|c|c|c|}
\hline$a$ & vitesse du son & $\mathrm{m} \cdot \mathrm{s}^{-1}$ \\
\hline$C_{\mathrm{f}}$ & coefficient de frottement & \\
\hline$C_{v, q}^{s}$ & chaleur spécifique à volume constant de l'espèce $s$ pour le mode interne d'énergie $q$ & $\mathrm{~J} . \mathrm{kg}^{-1} \cdot \mathrm{K}^{-1}$ \\
\hline$D_{s}$ & coefficient de diffusion de l'espèce $s$ & $\mathrm{~m} . \mathrm{s}^{-1}$ \\
\hline$E$ & énergie totale par unité de masse & ${\mathrm{J} . \mathrm{kg}^{-1}}^{-1}$ \\
\hline$e_{\mathrm{e}}$ & énergie électronique totale par unité de masse & $\mathrm{J}_{\mathrm{kg}}{ }^{-1}$ \\
\hline$e_{v_{m}}$ & énergie de vibration par unité de masse de la molécule $m$ & ${\mathrm{~J} . \mathrm{kg}^{-1}}^{-1}$ \\
\hline$h_{s}^{0}$ & enthalpie massique de formation de l'espèce $s$ & $\mathrm{~J}^{\mathrm{kg}} \mathrm{g}^{-1}$ \\
\hline$L_{\mathrm{e}}$ & nombre de Lewis & \\
\hline$M_{\mathrm{a}}$ & nombre de Mach & \\
\hline$\hat{M}_{s}$ & masse molaire de l'espèce $s$ & $\mathrm{~kg} \cdot \mathrm{mol}^{-1}$ \\
\hline$n m$ & nombre total de molécules & \\
\hline$n_{k}$ & normale extérieure à chaque face de la cellule $k$ & \\
\hline ns & nombre total d'espèces chimiques & \\
\hline$p$ & pression statique du mélange & $\mathrm{kg} \cdot \mathrm{m}^{-1} \cdot \mathrm{s}^{-2}$ \\
\hline$p_{s}$ & pression partielle de l'espèce $s$ & $\mathrm{~kg} \cdot \mathrm{m}^{-1} \cdot \mathrm{s}^{-2}$ \\
\hline$Q$ & flux de chaleur total & $\mathrm{W} \cdot \mathrm{m}^{-2}$ \\
\hline $\mathcal{R}$ & constante des gaz & J.mol ${ }^{-1} \cdot \mathrm{K}^{-1}$ \\
\hline$R$ & rayon de la calotte sphérique & $\mathrm{m}$ \\
\hline$R_{\mathrm{e}}$ & nombre de Reynolds & \\
\hline$T$ & température de translation-rotation du mélange & $\mathrm{K}$ \\
\hline$T_{\mathrm{e}}$ & température électron-électronique & $\mathrm{K}$ \\
\hline$T_{v_{m}}$ & température de vibration de la molécule $m$ & $\mathrm{~K}$ \\
\hline$t$ & temps & \\
\hline$U$ & vecteur conservatif & \\
\hline$u, v$ & composantes de la vitesse respectivement dans les directions $x$ et $y$ & $\mathrm{~m} \cdot \mathrm{s}^{-1}$ \\
\hline$Y_{s}$ & fraction massique de l'espèce $s$ & \\
\hline \multicolumn{3}{|c|}{ Symboles grecs } \\
\hline$\mu_{s}$ & viscosité dynamique de l'espèce $s$ & $\mathrm{~kg} \cdot \mathrm{m}^{-1} \cdot \mathrm{s}^{-1}$ \\
\hline$\Omega_{i, j}$ & terme source axisymétrique dans la cellule $i, j$ & \\
\hline$\lambda_{s}$ & conductivité thermique de l'espèce $s$ & $\mathrm{~W} \cdot \mathrm{m}^{-1} \cdot \mathrm{K}^{-1}$ \\
\hline$\rho$ & densité du mélange & $\mathrm{kg} \cdot \mathrm{m}^{-3}$ \\
\hline$\rho_{s}$ & densité de l'espèce $s$ & $\mathrm{~kg} \cdot \mathrm{m}^{-3}$ \\
\hline
\end{tabular}

et des phénomènes radiatifs. Il est donc nécessaire de tenir compte dans la modélisation de l'ensemble de ces phénomènes.

Le vol à grande vitesse se caractérise par des phénomènes physiques moléculaires couvrant une large gamme de nombres de Mach, de Reynolds, de Knudsen et de Damköhler. Ainsi la simulation expérimentale dans les installations au sol, est sévèrement limitée en raison de l'impossibilité de respecter une similitude totale des conditions de vol. Cette similitude nécessite simultanément la reproduction correcte de la densité du gaz, de la vitesse de vol et de la maquette. Par ailleurs, la simulation en vol est difficilement réalisable $\mathrm{du}$ point de vue de l'instrumentation du prototype et elle reste toujours très onéreuse. Le développement des modèles théoriques et des techniques modernes de simulation numérique, conjointement validés sur le plan expérimental, sont des moyens efficaces pour progresser dans ce domaine. Il est désormais nécessaire de s'attacher à résoudre plusieurs problèmes sur le plan de la modélisation avec validation par l'expérience. Les travaux réalisés ces dernières années [1-3] ont permis de progresser dans la compréhension et la modélisation des écoulements réactifs en déséquilibre thermochimique.

L'objectif de la présente étude est la prise en compte de l'ionisation, du non-équilibre chimique, vibrationnel et électronique afin d'étendre les travaux existants et de mieux prédire les flux de chaleur autour des engins de rentrée. Le gaz utilisé est de l'air faiblement ionisé, constitué de 7 espèces chimiques $(\mathrm{O}, \mathrm{N}, \mathrm{NO}$, $\mathrm{O}_{2}, \mathrm{~N}_{2}, \mathrm{NO}^{+}, \mathrm{e}^{-}$) et est thermiquement caractérisé par 4 températures $\left(T, T_{v_{\mathrm{O}_{2}}}, T_{v_{\mathrm{N}_{2}}}, T_{\mathrm{e}}\right)$. Les molécules $\mathrm{O}_{2}$, $\mathrm{N}_{2}$ sont en déséquilibre vibrationnel tandis que $\mathrm{NO}$ et $\mathrm{NO}^{+}$sont supposées en équilibre thermique avec le 
mode de translation $T$. La cinétique chimique utilisée [4] est à 24 réactions élémentaires. Le couplage vibrationdissociation (CVD) suit le modèle de Park [4]. Le couplage Vibration-Vibration (V-V) utilisé est celui développé par Candler [5] dans lequel les probabilités de collisions proviennent des données expérimentales de Taylor et al. [6] et ont été mis récemment sous forme exponentielle par Park et al. [7]. Le couplage Translation-Électronique (T-E) est donné par Appleton et al. [8] et le couplage VibrationÉlectronique (V-E) est modélisé d'après la relation de Landau-Teller [9].

La simulation est réalisée à partir du code de calcul robuste et efficace CARBUR intensivement validé [10-12] pour des écoulements visqueux et axisymétriques. Ce code multi-blocks est basé sur un schéma de type volumes finis, dans lequel sont inclus les processus d'ionisation, de non-équilibre thermochimique et des différents couplages existants. Les calculs menés pour des nombres de Mach compris entre 15 et 18 sont comparés à d'autres simulations numériques et aux données expérimentales.

\section{2 Équations et relations}

Le système d'équations de Navier-Stokes résolu dans ce travail est constitué de ns équations de conservation de masse des espèces chimiques, de deux équations de quantité de mouvement dans les directions $x$ et $y$, d'une équation de conservation d'énergie totale, de $n s v$ équations de relaxation vibrationnelle pour $n s v$ molécules pris en non-équilibre vibrationnel et enfin d'une équation de relaxation électronique [13]. Il s'écrit sous la forme condensée :

$$
\frac{\partial U}{\partial t}+\nabla \cdot F(U)=\Omega
$$

où $U=\left(\rho Y_{1}, \ldots, \rho Y_{n s}, \rho u, \rho v, \rho e, \rho e_{v_{1}}, \ldots, \rho e_{v_{n m v}}, \rho e_{\mathrm{e}}\right)^{T}$ est le vecteur des variables conservatives, $F=F_{\mathrm{c}}+F_{\mathrm{v}}$ la somme des flux convectifs $F_{\mathrm{c}}$ et visqueux $F_{\mathrm{v}}$ et $\Omega$ est le terme source. Avec :

$$
\begin{aligned}
& F_{\mathrm{c}_{x}}=\left[\rho_{i} u, \rho u^{2}+p, \rho u v,(\rho e+p) u, \rho e_{v_{m}} u, \rho_{e_{\mathrm{e}}} u\right]^{T} \\
& F_{v_{x}}=\left[\rho_{i} V_{x_{i}}, \tau_{x x}, \tau_{x y}, u \tau_{x x}+v \tau_{x y}+q_{x}, q_{v_{m} x}, q_{e x}\right]^{T} \\
& \Omega=\left[\omega_{i}, 0,0,0, \Omega_{r v_{m}}, \Omega_{r e_{\mathrm{e}}}\right]^{T} \\
& \Omega_{r v_{m}}=Q_{T-v_{m}}+Q_{v-v_{m}}+Q_{e-v_{m}} \\
& \Omega_{r e_{e}}=Q_{T-e}+\sum_{m} Q_{e-v_{m}}-P_{\mathrm{e}}\left(\frac{\partial u}{\partial x}+\frac{\partial v}{\partial y}\right)+\omega_{e l}
\end{aligned}
$$

L'énergie de vibration et électronique par unité de masse sont données par la distribution de Boltzmann à la température caractéristique de chaque mode [9] Seuls les niveaux électroniques ayant des températures caractéristiques inférieures à $50000 \mathrm{~K}$ sont considérés dans ce travail.

L'équation d'état du gaz permet de fermer le système d'équations. On suppose que chaque espèce se comporte comme un gaz parfait et la pression du mélange est donnée par la loi de Dalton :

$$
p=\sum_{s \neq e} \rho_{s} \mathcal{R} T / \hat{M}_{s}+p_{e}
$$

La température de translation $T$ est obtenue par une méthode itérative à partir de la relation de l'énergie totale du mélange par unité de volume

$$
\begin{array}{r}
\rho E=\sum_{s \neq e} \rho_{s} C_{v, t r}^{s} T+\frac{1}{2} \sum_{s} \rho_{s} u_{s}^{2}+\sum_{m=1}^{n m} \rho_{m} e_{v_{m}}+\rho e_{\mathrm{e}} \\
+\sum_{s}^{n s} \rho_{s} h_{s}^{0}
\end{array}
$$

Dans le calcul des flux visqueux, le coefficient de viscosité est déterminé à partir des viscosités de chacune des espèces $\mu_{s}$ donnée par la relation de Blottner et al. [14]. La conductivité thermique résultante de chaque espèce $\lambda_{s}$ est donnée par la relation d'Euken [9]. La viscosité et la conductivité du mélange sont déterminées à partir de la formule semi-empirique de Wilke [15].

Le flux de chaleur total $Q$ est la résultante des flux de conduction pour le mode de translation, de vibration, d'électronique et de diffusion d'énergie totale. Il s'écrit sous une forme plus pratique à manipuler comme étant [12] :

$$
\begin{aligned}
Q=-\frac{\lambda_{t r}^{\prime}}{C_{p f}^{\prime}}[ & \nabla h+\left(L_{e}^{\prime}-1\right) \\
& \left.\times\left(\sum_{s=1}^{N S} h_{s} \nabla Y_{s}+\sum_{s=1}^{N S V} Y_{s} \nabla e_{v_{s}}+\nabla e_{e}\right)\right]
\end{aligned}
$$

La barodiffusion et l'effet Soret étant négligés, le flux de diffusion est donné par une approximation de premier ordre qui conduit à la loi de Fick [9]. Quand l'écoulement est faiblement ionisé, le coefficient de diffusion des ions et des électrons est modélisé par la formulation de la diffusion ambipolaire de Ramsaw et al. [16]. Le coefficient de diffusion $D$ est calculé à partir du nombre de Lewis supposé constant et égal à 1,2 ; ce qui permet de s'affranchir d'une étape de calcul des coefficients de diffusion polynaire souvent très coûteuse. L'hypothèse de l'électroneutralité locale dans tout l'écoulement est faite.

\section{Méthodes numériques}

La résolution numérique du système (1) nécessite l'utilisation de méthodes numériques suffisamment robustes, précises et bien adaptées. La méthode de volumes finis d'ordre élevé vérifiant les propriétés TVD est utilisée. L'un des atouts de la méthode réside dans l'utilisation de la formulation intégrale qui facilite le traitement des discontinuités et traite de manière cohérente la topologie du domaine d'intégration. 
Pour un volume de contrôle arbitraire $V_{i j}$, délimité par la surface $\partial V_{i j}$, toutes les équations peuvent se mettre sous la forme intégrale suivante :

$$
\frac{\partial}{\partial t} \int_{V_{i, j}} U \mathrm{~d} v+\int_{\partial V_{i, j}} F(U) n \mathrm{~d} \partial V=\int_{V_{i, j}} \Omega \mathrm{d} v
$$

où le vecteur conservatif $U$ est constant dans le volume $V_{i j}, F(U)$ est la somme des flux convectifs et visqueux, $n$ est le vecteur unitaire normal à la surface $\partial V_{i, j}$ et $\Omega$ est le terme source linéarisé par rapport au vecteur conservatif. Les flux visqueux $F_{\mathrm{v}}$ sont discrétisés à l'aide des différences finies centrées. Les flux convectifs $F_{\mathrm{c}}(U) \cdot n$ à l'interface de chaque cellule sont calculés par une approche FDS (Flux-Difference-Splitting) utilisant la solution locale d'un problème de Riemann. L'utilisation de l'approche MUSCL-Hancock [17] permet d'obtenir une précision d'ordre deux en espace. Le solveur excat de Riemann et la fonction limiteur Mimmod sont utilisés pour l'évaluation des flux convectifs.

Le prédicteur-correcteur explicite en temps permet d'obtenir l'état stationnaire après convergence de la formulation instationnaire du système d'équations discrétisées. Le schéma utilisé a une précision de second ordre en temps et en espace.

Le déséquilibre électronique entraîne une correction sur la vitesse appropriée du son utile à la décomposition des flux convectifs [13] :

$$
a^{2}=\tilde{\gamma}\left(\frac{p}{\rho}\right)+(\tilde{\gamma}-1)\left(\frac{T}{T_{\mathrm{e}}}-1\right) \frac{p_{\mathrm{e}}}{\rho}
$$

Cette expression se réduit à la relation classique dans le cas où l'hypothèse d'une température unique de translation est faite $\left(T \equiv T_{\mathrm{e}}\right)$.

\section{Conditions aux limites et initiales}

\section{Conditions aux limites}

Les conditions d'adhérence de vitesse et de température fixée sont imposées à la paroi. La paroi supposée non poreuse et chimiquement noncatalytique est considérée en équilibre thermodynamique à la température de paroi $\left(T_{\mathrm{p}}\right)$. L'écoulement infini amont est hypersonique et supersonique en sortie. Le long de l'axe de symétrie, des conditions de réflection ou de glissement sont utilisées.

\section{Conditions initiales}

Les conditions infini amont de calcul sont regroupées dans le tableau 1. L'écoulement infini amont est de l'air dont les fractions massiques sont initialement constituées de $76,699 \%$ de $\mathrm{N}_{2}$ et de $23,301 \%$ pour $\mathrm{O}_{2}$. La distribution de points suivant $x$ et $y$ du domaine de calcul suit une progression géométrique qui permet de densifier le maillage
Tableau 1. Conditions infini amont et de calcul.

\begin{tabular}{lccc}
\hline Mach & 15,35 & 16 & 18 \\
Altitude, $(\mathrm{km})$ & 35 & 37 & 37 \\
Rayon $R,(\mathrm{~m})$ & $6,35 \times 10^{-3}$ & $6,6 \times 10^{-3}$ & $6,6 \times 10^{-3}$ \\
$U_{\infty},\left({\left.\mathrm{m} . \mathrm{s}^{-1}\right)}\right.$ & 5263 & 5104,44 & 5742,49 \\
$P_{\infty},\left(\mathrm{P}_{a}\right)$ & 664 & 432,2 & 432,2 \\
$T_{\infty},(\mathrm{K})$ & 293 & 252 & 252 \\
$T_{p},(\mathrm{~K})$ & 1000 & 555,5 & 555,5 \\
$R_{e_{\infty}}$ & 14605 & 11413 & 12840 \\
$K_{n_{\infty}}$ & $1,55 \times 10^{-3}$ & $2,0828 \times 10^{-3}$ & $4,2824 \times 10^{-3}$ \\
Expérience & Ref. $[18]$ & Ref. $[25]$ & Ref. $[26]$ \\
Nœuds IM & 50 & 50 & 60 \\
Nouds JM & 50 & 50 & 60 \\
$\Delta x_{\text {min }}(\mathrm{m})$ & $4,1936 \times 10^{-6}$ & $3,229 \times 10^{-6}$ & $1,4231 \times 10^{-6}$ \\
$\Delta y_{\text {min }}(\mathrm{m})$ & $7,2055 \times 10^{-5}$ & $7,48 \times 10^{-5}$ & $4,6432 \times 10^{-5}$ \\
\hline
\end{tabular}

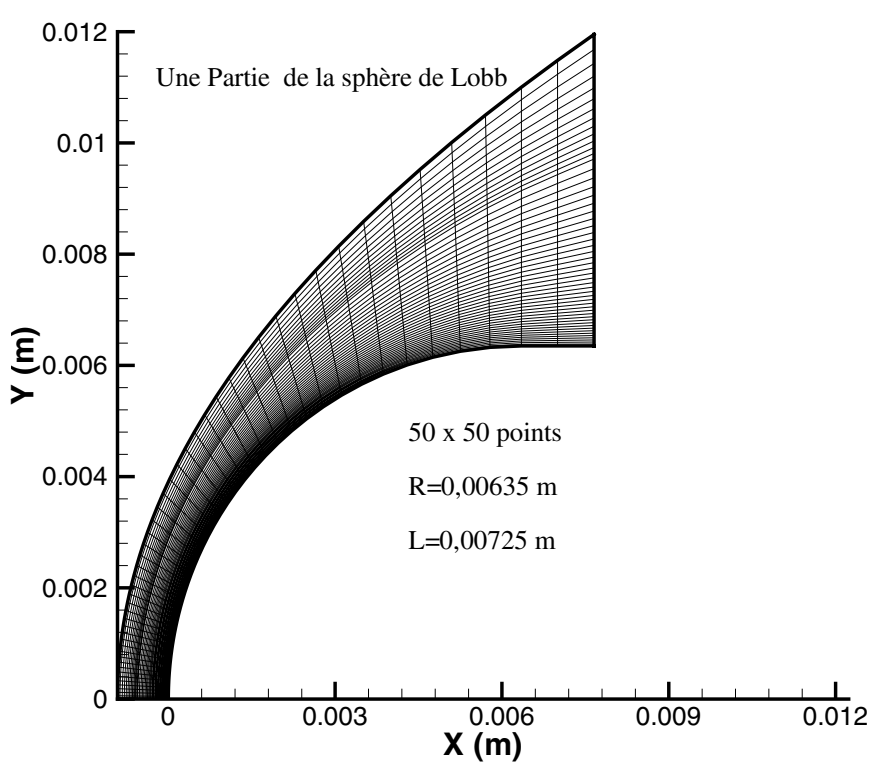

Fig. 1. Maillage de calcul, sphère Lobb.

dans les zones de l'écoulement où existent des forts gradients comme le montre la figure 1 . Le nombre de points ainsi que les pas minimaux sont également mentionnés dans le tableau 1. IM et JM représentent respectivement le maximum de points suivant $x$ et $y$.

Le pas de temps est obtenu à partir du critère de stabilité du Courant-Friedrichs-Lewy (CFL). Les valeurs de $0,1 \leq C F L \leq 0,8$ utilisées permettent d'obtenir un schéma stable dans les différents cas étudiés.

\section{Résultats et discussions}

Les résultats sont obtenus pour des sphère-cylindre placées dans un écoulement hypersonique en nonéquilibre thermochimique sans incidence. Les différentes influences des effets de déséquilibre (chimique, vibrationnel, électronique) sont examinées sur la structure de 


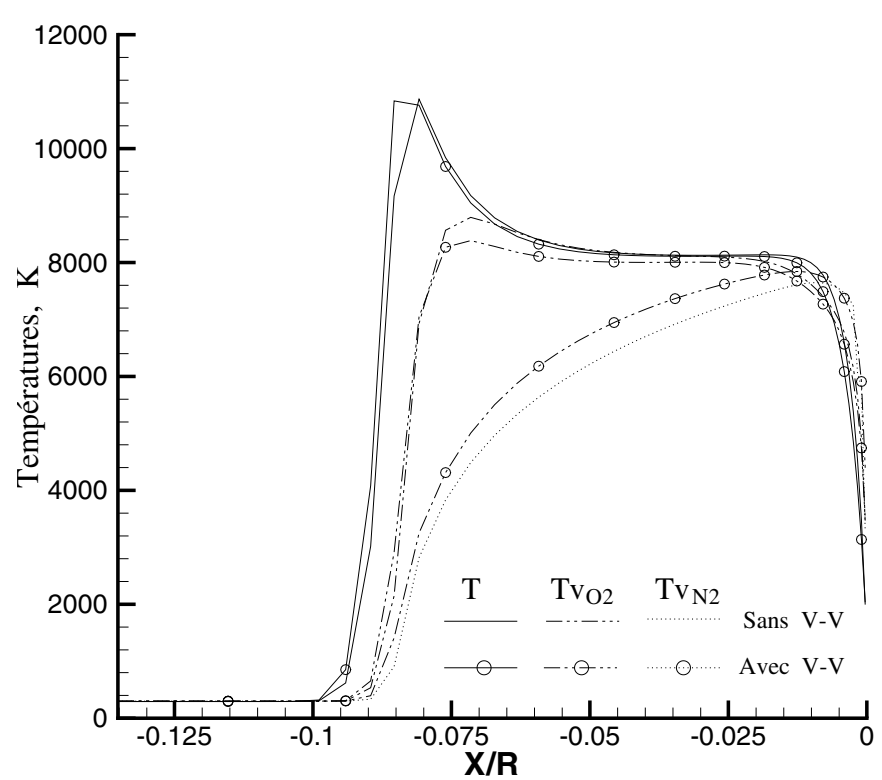

Fig. 2. Températures le long de la ligne d'arrêt : Mach 15,3.

l'écoulement. Ces résultats sont validés avec des données expérimentales et d'autres prédictions numériques.

\subsection{Sphère de Lobb : Mach 15,3}

Lobb [18] a effectué des tirs de sphères non catalytiques à Mach 15,35 dans de l'air porté à $293 \mathrm{~K}$ sous une pression de $664 P_{\mathrm{a}}$. Les différentes mesures ont permis d'évaluer de manière expérimentale la distance de détachement de l'onde de choc par rapport à l'obstacle. Des travaux numériques ont également été menés sur ce cas par plusieurs auteurs [2,19-22]. L'intérêt de cette étude est de comparer les résultats du présent modèle avec ceux existants.

La figure 2 montre de manière comparative la distribution des températures $\left(T, T_{v_{\mathrm{O}_{2}}}, T_{v_{\mathrm{N}_{2}}}\right)$ sur la ligne d'arrêt pour un calcul mené avec la chimie à 5 espèces $\left(\mathrm{O}, \mathrm{N}, \mathrm{NO}, \mathrm{O}_{2}, \mathrm{~N}_{2}\right)$. Les énergies de vibration s'excitent après l'énergie de transrotation, la région de déséquilibre vibrationnel étant clairement visible pour les molécules d'oxygène et d'azote avec une distribution différente de températures. Au voisinage de la paroi qui correspond à la couche limite visqueuse, tous les modes d'énergie relaxent vers un équilibre à la température de paroi. Sous l'influence du couplage V-V la température de translation reste presque insensible tandis que les températures de vibration tendent à se rapprocher. On note un transfert d'énergie de vibration de $\mathrm{O}_{2}$ vers $\mathrm{N}_{2}$, qui se traduit par une diminution de l'ordre de $4 \%$ de $T_{v_{\mathrm{O}_{2}}}$ dans la zone de relaxation et une augmentation de $T_{v_{\mathrm{N}_{2}}}$ dans le même ordre de grandeur. Le degré de déséquilibre chimique est observé sur la figure 3 avec une présentation de la distribution des fractions massiques. Derrière l'onde de choc, les molécules d'oxygène sont dissociées à plus de $95 \%$ et celles d'azote à plus de $10 \%$. Les fractions massiques des différentes espèces restent quasi identiques à celle obtenue avec le couplage $\mathrm{V}-\mathrm{V}$. On note une prédominance

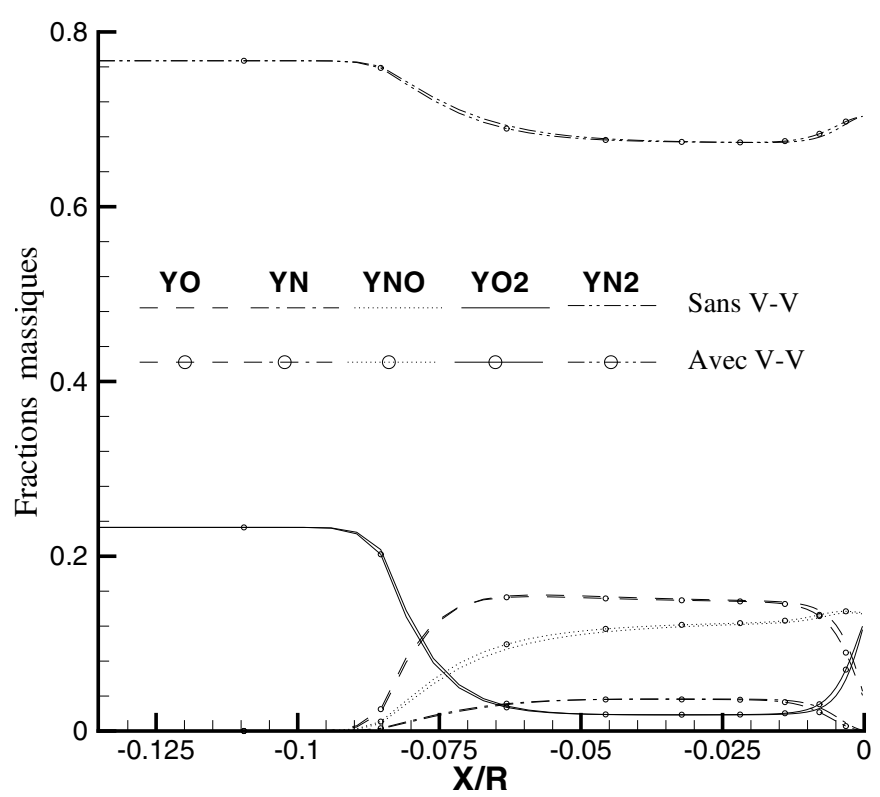

Fig. 3. Fractions massiques le long de la ligne d'arrêt : Mach 15,3.

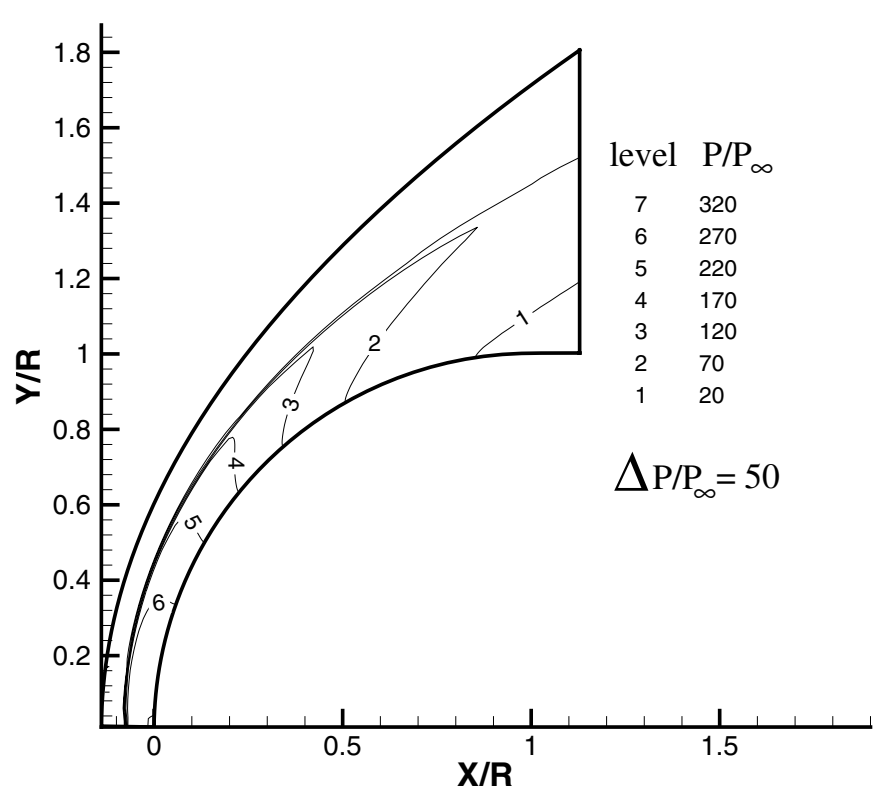

Fig. 4. Isovaleurs de la pression : Mach 15,3.

des réactions de recombinaison au voisinage de la paroi, du fait de la paroi gardée relativement froide à la température constante de $1000 \mathrm{~K}$.

Un second calcul est effectué avec un écoulement faiblement ionisé à 7 espèces chimiques $\left(\mathrm{O}, \mathrm{N}, \mathrm{NO}, \mathrm{O}_{2}, \mathrm{~N}_{2}\right.$, $\mathrm{NO}^{+}, \mathrm{e}^{-}$) dans lequel la relaxation électronique est pris en compte. Le champ de pression obtenu est représenté par les isopressions à la figure 4. On distingue une zone de forte compression et une zone de détente. La valeur maximale de la pression sur la ligne d'arrêt est considérée comme étant la position du choc. Les différentes valeurs prédites sont comparées dans le tableau 2. Le présent calcul conduit à une légère diminution de l'ordre de $3 \%$ entre la prise en compte du déséquilibre électronique et le 
Tableau 2. Comparaison de la position du choc sur la ligne d'arrêt : Mach 15,3.

\begin{tabular}{ll}
\hline Auteurs & $\mathrm{X} / \mathrm{R},(\%)$ \\
\hline Candler [22] & 10,5 \\
Flament [19] & 9,42 \\
Thivet [19] & 8,84 \\
Séror CVDEV [2] & 8,77 \\
Schall CVDV $(U=\infty)[20]$ & 8,67 \\
Tam \& Campbell [21] & 8,2 \\
Présent (figé) & 12,59 \\
Présent (avec CVD) & 8,66 \\
Présent (avec CVD + V-V) & 8,65 \\
Présent (avec électronique) & 8,36 \\
Expérience - Lobb [18] & $8,7 \pm 0,5$ \\
\hline
\end{tabular}

couplage V-V. Les schémas numériques utilisés par Candler [22] et Schall [20] sont identiques, mais le maillage de calcul reste grossier chez Candler. Le calcul de Flament est limité au couplage CVD. Les résultats de Thivet [19] prennent simultanément en compte les couplages CVD et V-V. Séror [2] tient compte du couplage CVDEV. Tam et al. [21] prend en compte l'ionisation, avec un modèle thermique simplifié à 2 températures $\left(T, T_{\mathrm{v}}\right)$. Les divergences observées peuvent être attribuées au modèle et à la démarche numérique.

La figure 5 montre les évolutions du coefficient de frottement le long de la paroi. La prise en compte du déséquilibre électronique s'accompagne d'une baisse de l'ordre de $5 \%$ du maxima. Les résultats de Séror [2] sont obtenus à partir de l'approche déficitaire des couches limites réactives et ceux de Joly et al. [23] à partir du code CELHYO développé à l'ONERA. Les deux codes ne prennent pas en compte la relaxation électronique, et utilisent une cinétique chimique de l'air à 5 espèces. Le flux de chaleur obtenu dans les mêmes conditions est également comparé à la figure 6 . On remarque que négliger la contribution électronique sous-évalue de l'ordre de $4 \%$ le flux de chaleur au niveau du point d'arrêt. Une différence de flux de l'ordre de $11 \%$ est observée avec les résultats de Séror au niveau du point d'arrêt. La cinétique chimique utilisée par Séror est celle de Gardiner avec un modèle de Baulch et al. [24] pour les réactions d'échange. Les écarts entre les différentes valeurs se réduisent et deviennent presque identiques au fur et à mesure que l'on approche la zone de détente où les transferts thermiques sont moins intenses.

\subsection{Sphère-cylindre de Rose}

L'étude de ce cas est motivée par l'existence de mesures expérimentales de flux de chaleur laminaire au point d'arrêt obtenues par Rose et al. [25, 26]. Les valeurs obtenues dans un écoulement d'air ont ensuite été comparées à la prévision théorique du modèle simple de diffusion binaire de l'azote diatomique partiellement

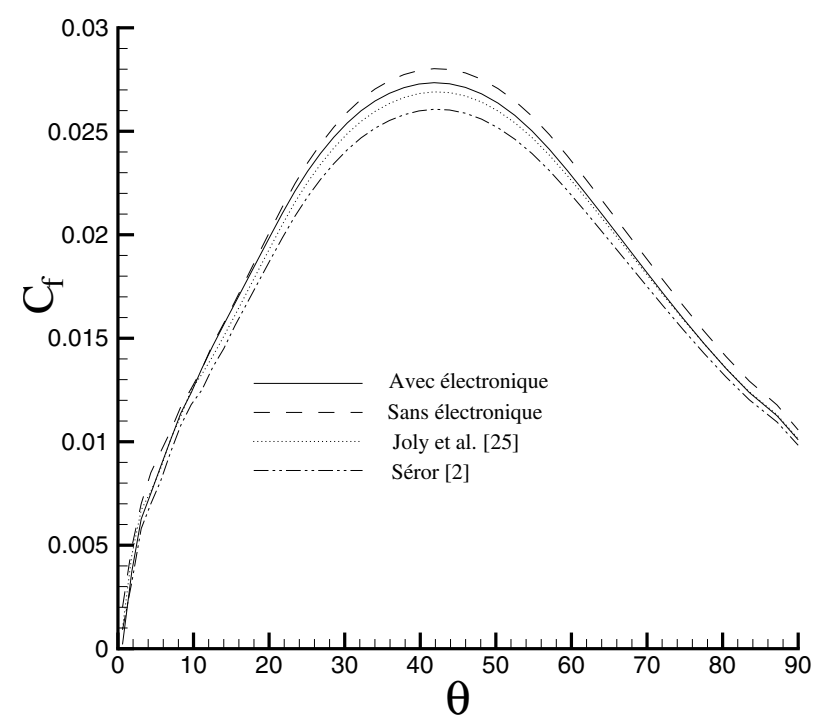

Fig. 5. Comparaison, coefficient de frottement le long de la paroi : Mach 15,3.

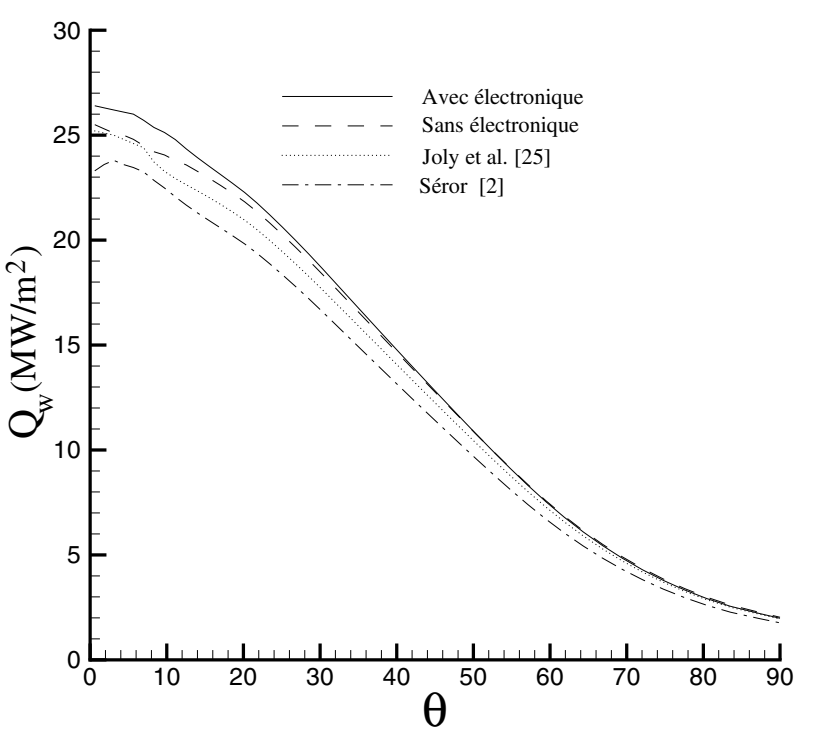

Fig. 6. Comparaison, valeurs du flux de chaleur pariétal : Mach 15,3.

dissocié de Fay et Riddell [27]. Des résultats de simulations numériques ont été publiés sur cette sphère par Josyula et al. [28], Schall et al. [20].

Deux cas de vol à la même altitude sont simulés pour cette sphère : les cas Mach 16 et Mach 18 dont les conditions infini amont sont regroupées dans le tableau 1.

\section{Cas Mach 16}

Les évolutions des températures et des fractions massiques le long de la ligne d'arrêt représentées sur les figures 7 et 8 confirment les observations faites sur le cas Mach 15,35. On note une diminution du maximum de $T_{v_{\mathrm{O}_{2}}}$ de l'ordre de $4,5 \%$ derrière l'onde de choc du fait du couplage $\mathrm{V}-\mathrm{V}$. Ce couplage affecte légèrement les 


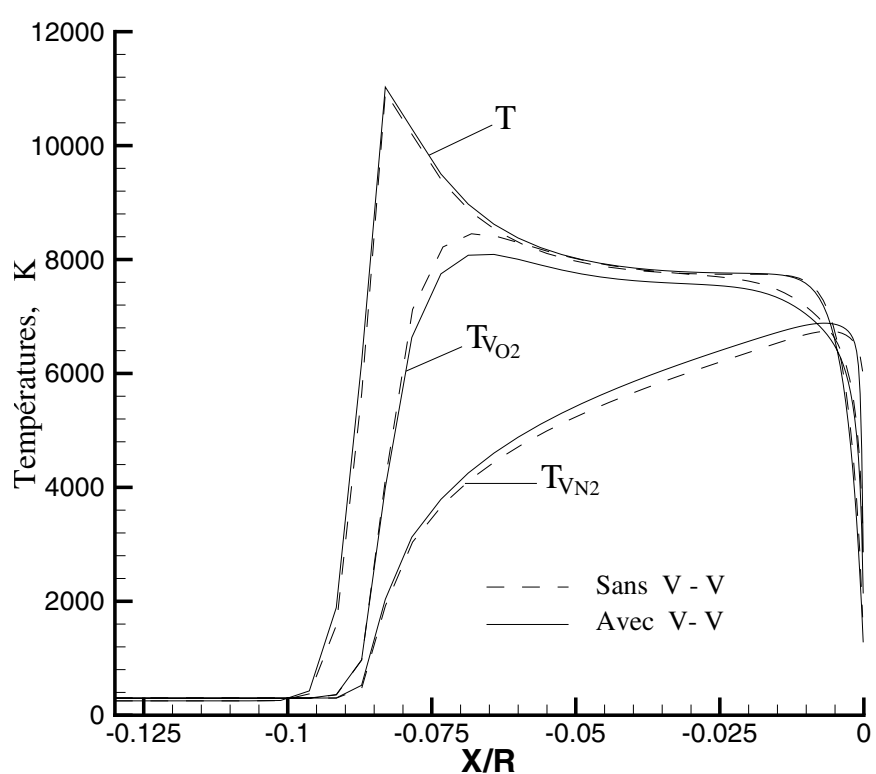

Fig. 7. Températures le long de la ligne de d'arrêt : Mach 16.

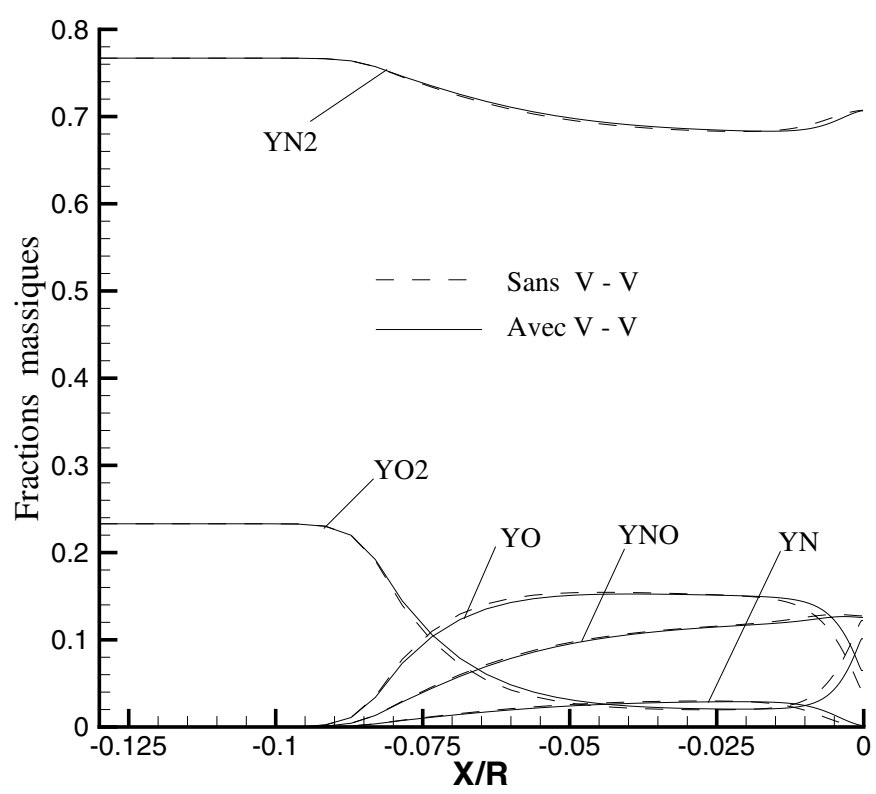

Fig. 8. Fractions massiques le long de la ligne de d'arrêt : Mach 16.

réactions chimiques au voisinage de la paroi et induit une influence sur le flux de chaleur comme le montre la figure 9. La dissociation des molécules est ralentie et les recombinaisons des atomes qui sont des réactions exothermiques se mettent en place au voisinage de la paroi à $T_{\mathrm{p}}=555,5 \mathrm{~K}$. Ceci entraîne une augmentation de flux de chaleur de l'ordre de 3,84 \% au niveau du point d'arrêt. Les valeurs de flux de chaleur au point d'arrêt du présent modèle sont comparées avec celles obtenues par Schall et al. [20] (31 MW.m $\left.{ }^{-2}\right)$, Josyula et al. [28] $\left(22 \mathrm{MW} . \mathrm{m}^{-2}\right)$, la mesure $\left(29,8 \mathrm{MW} \cdot \mathrm{m}^{-2}\right)$. L'écart observé avec la valeur de Josyula peut être dû à la cinétique chimique. Les réactions de recombinaison ne sont pas identiques au voisinage de la paroi.

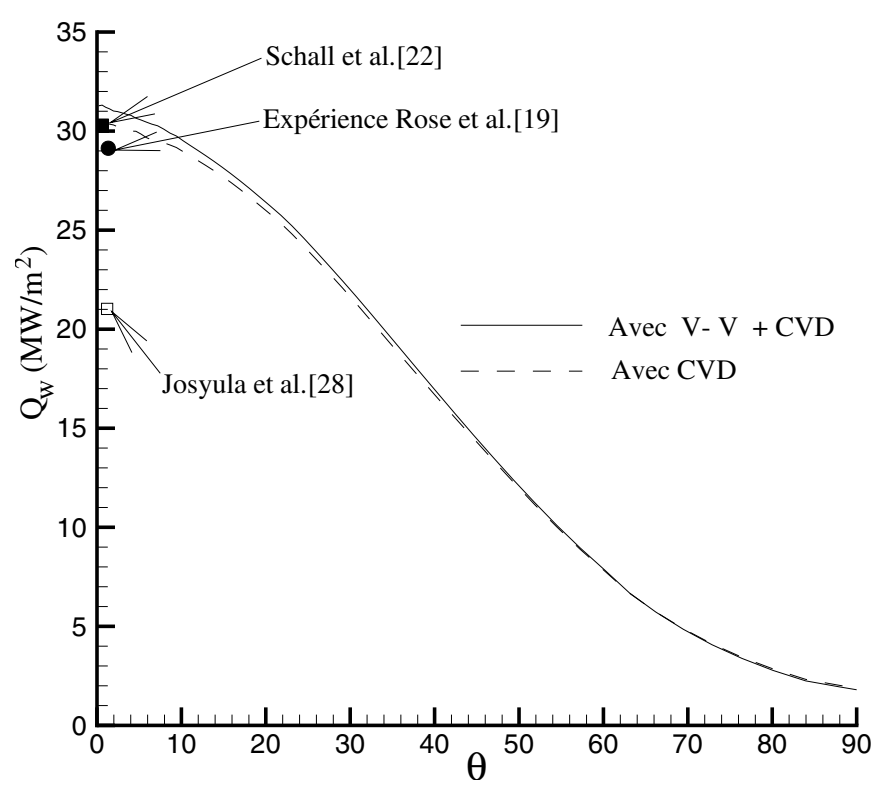

Fig. 9. Flux de chaleur pariétal : Mach 16.

Tableau 3. Positions du choc sur la ligne d'arrêt : Mach 18.

\begin{tabular}{ll}
\hline Auteurs & $\mathrm{X} / \mathrm{R},(\%)$ \\
\hline Josyula et al. [28] & 9,24 \\
Schall [20] (CVDV, $U=\infty)$ & 8,60 \\
Présent (figé) & 11,48 \\
Présent (CVD) & 8,21 \\
Présent (CVD + V-V) & 8,19 \\
Présent (électronique) & 8,12 \\
\hline
\end{tabular}

Cas Mach 18

La position de l'onde de choc obtenue sur la ligne d'arrêt pour les différents calculs est portée dans le tableau 3. Le cas figé correspond à un calcul sans déséquilibre chimique. Schall et al. [20] utilise un maillage à 2500 points avec un raffinement des mailles au niveau de l'onde de choc comme c'est le cas dans la présente étude. Josyula et al. [28] utilise le solveur de Roe avec une précision d'ordre 1 sur un maillage à 2000 points qui reste grossier au niveau de l'onde de choc. Les différents effets de non-équilibre et de couplages repositionnent l'onde de choc.

L'influence de la correction de la vitesse du son sur la structure de l'écoulement est examinée sur les figures 10 et 11 avec une coupe sur la ligne d'arrêt des températures et des fractions massiques. La répartition de l'énergie thermique sur les différents modes internes permet de se rapprocher de la réalité physique. Il est judicieux de tenir compte de cette correction qui permet d'obtenir une meilleure approximation de la vitesse locale de propagation de l'information. La fraction massique des électrons n'est que très légèrement affectée par cette correction du fait que la réaction de production des ions et électrons est en partie contrôlée par $T_{\mathrm{e}}$ à travers le couplage CVD. 


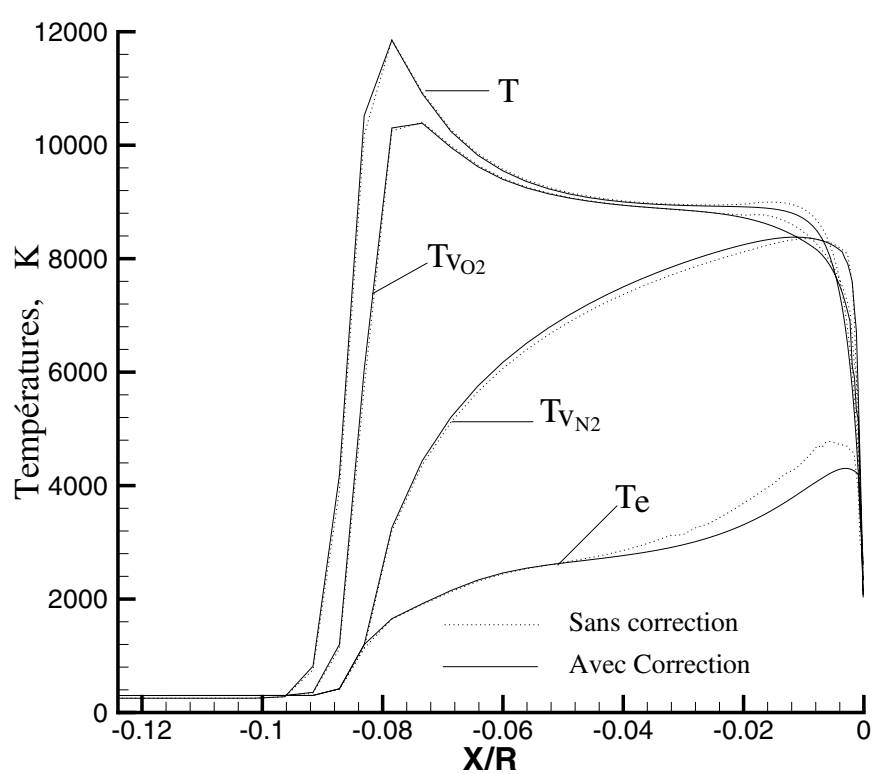

Fig. 10. Influence de la vitesse modifiée du son sur les températures : Mach 18.

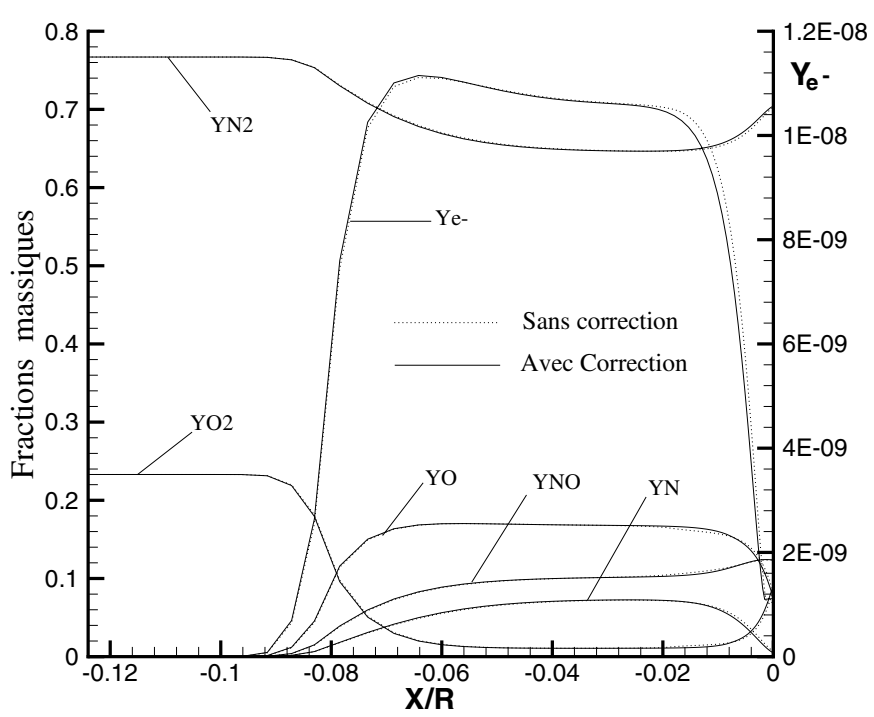

Fig. 11. Influence de la vitesse modifiée du son sur les fractions massiques : Mach 18.

Les évolutions de flux de chaleur sont représentées sur la figure 12. La prise en compte de l'ionisation et de la relaxation électronique conduit à une augmentation du flux de chaleur de l'ordre de 3,4 \% au niveau du point d'arrêt. Les valeurs de Schall et al. [20] (47 MW.m ${ }^{-2}$ ), de Josyula et al. [28] (29,5 MW.m $\left.{ }^{-2}\right)$, de Fay et al. [27] (45 MW.m ${ }^{-2}$ ) et la mesure de Rose et al. [26] (46 MW.m ${ }^{-2}$ ) sont également reportées. La recombinaison de $\mathrm{N}_{2}$ et $\mathrm{O}_{2}$ induit une augmentation des énergies de vibration dans la couche limite et de leurs gradients à la paroi. La valeur prédite par l'expression théorique de Fay et al. [27] est obtenue pour un écoulement laminaire réactif avec un nombre de Lewis pris égal à 2,0. Les résultats provenant du présent calcul sont légérement plus bas que l'expérience. Cette légère différence peut être attribuée

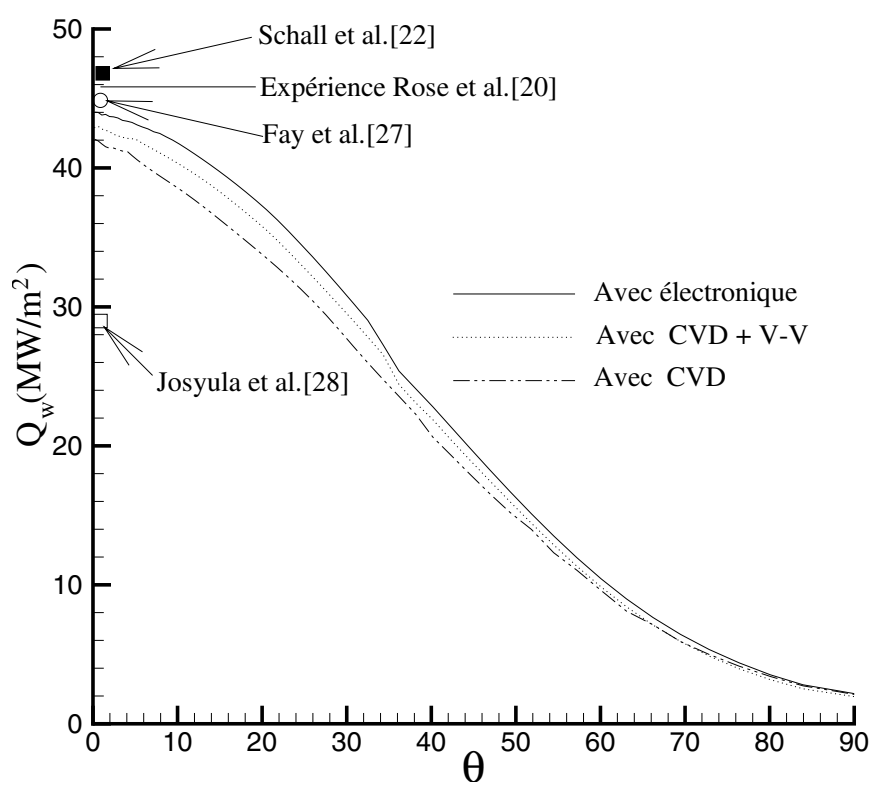

Fig. 12. Flux de chaleur le long de la paroi : Mach 18.

aux effets radiatifs négligés, qui auraient tendance en haute enthalpie d'accroître le flux.

\section{Conclusion}

Le code CARBUR a été étendu à la simulation numérique des écoulements faiblement ionisés en nonéquilibre incluant les processus de déséquilibre vibrationel et électronique. Le nouveau code obtenu a permis de calculer des écoulements 2D axisymétriques autour des corps de rentrée atmosphérique. Les effets des différents phénomènes thermochimiques ont permis de dégager leurs influences sur les paramètres aérothermodynamiques. La prise en compte du déséquilibre électronique modifie la cinétique chimique et peut affecter jusqu'à $4 \%$ la position du choc pour les cas étudiés. Négliger la relaxation électronique conduit à une surévaluation des activités chimiques et à une sous-estimation du flux de chaleur au niveau de la paroi.

Pour une étude plus complète, il est nécessaire de prendre en compte l'influence des conditions de catalyticité, de glissement à la paroi et des phénomènes d'émission radiative importants à très haute température. Sur le plan théorique, une description plus précise des termes de transport apporterait une amélioration au modèle.

\section{Références}

[1] J. William, Étude des processus physico-chimiques dans les écoulements détendus à hautes enthalpies : Application à la soufflerie à Arc F4, Thèse de doctorat, Université de Provence, IUSTI, 1999 
[2] S. Séror, Extension de l'approche déficitaire pour le calcul des couches limites hypersoniques en déséquilibre chimique et vibrationnel, Modélisation du couplage vibration/réactions d'échange, Thèse de doctorat, École nationale supérieure de l'aéronautique et de l'espace, 1997

[3] C. Flament, Écoulement de fluide visqueux en déséquilibre chimique et vibrationnel, modélisation thermo-chimique, Thèse de doctorat, Université de Paris VI, 1990

[4] C. Park, Nonequilibrium Hypersonic Aerothermodynamics, John Wiley and Sons, New York, 1990

[5] G.V. Candler, R.W. MacCormarck, The computation of hypersonic ionized flows in chemical and thermal nonequilibrium, AIAA Paper 88-0511, 1988

[6] R. Taylor, M. Camac, M. Feinberg, Measurement of vibration-vibration coupling in gas mixtures, in proceeding of the 11th international symposium on combustion, Pittsburg, PA, 1967, pp. 49-65

[7] C. Park, S.H. Lee, Validation of multitemperature nozzle flow code NOZNT, in AIAA 28nd Thermophysics Conference, Orlando, FI. AIAA Paper 93-2862, July 1993

[8] J.P. Appleton, K.N.C. Bray, The conservation equations for a nonequilibrium plasma, J. Fluid Mech 20 (1964) 659-672

[9] W.G. Vincenti, C.H. Jr. Kruger, Introduction to physical Gas Dynamics, Krieger, FL, 1965

[10] Y. Burtschell, M. Cardoso, D.E. Zeitoun, Numerical analysis of reducing driver gas contamination in impulse shock tunnels, AIAA J. 39 (2001) 2357-2365

[11] L. Meister, Y. Burtschell, D.E. Zeitoun, Étude numérique d'un écoulement réactif dans une tuyère surdétendue : influence de la température de paroi et de l'altitude, Mécanique \& Industries 4 (2003) 339-346

[12] G. Tchuen, Y. Burtschell, D.E. Zeitoun, Numerical prediction of weakly ionized high enthalpy flow in thermochemical nonequilibrium, AIAA Paper 04-2462, June 2004

[13] G. Tchuen, Modélisation et simulation numérique des écoulements à haute enthalpie : influence du déséquilibre électronique, Thèse de doctorat de l'Université de Provence, France, 2003

[14] F.G. Blottner, M. Johnson, M. Ellis, Chemically reacting viscous flow program for multi-component gas mixtures, Sandia Laboratories, Albuquerque, NM, Rept. Sc-RR-70754, Dec. 1971.

[15] C.R. Wilke, A viscosity Equation for Gas Mixture, J. Chem. Phys. 18 (1950) 517-519
[16] J.D. Ramsaw, C.H. Chang, Ambipolar diffusion in two temperature multicomponent plasma, Chem. Plasma process 13 (1993) 489-498

[17] E.F. Toro, Riemann solvers and numerical methods for fluid dynamics, A pratical introduction, 2nd edition, Springer 1999

[18] K. Lobb, Experimental measurement of shock detachment distance on sphere fired in air at hypervelocities, Proceeding of the AGARD-NATO in The high temperature aspect of hypersonic flow, Specialists Meeting, 3-6 April 1962, Ed. W.C. Nelson, Pergamon Press

[19] F. Thivet, Modélisation et calcul d'écoulements hypersoniques en déséquilibre chimique et thermodynamique, Thèse, École centrale de Paris, 1992

[20] E. Schall, Y. Burtschell, D.E. Zeitoun, Numerical study of nonequilibrium process in high enthalpy flow, Int. J. Num. Meth. for Heat and Fluid Flow 5 (1995) $271-281$

[21] L.T. Tam, M.Y. An, C.H. Campbell, Nonequilibrium hypersonic flow computation for weakly ionized air and comparison with flight data, AIAA paper 93-3195, AIAA 24th Plasmadynamics and Lasers Conference, July 6-9, 1993, Orlando, Florida

[22] G.V. Candler, Computation of weakly ionized hypersonic flow in thermochemical nonequilibrium, Ph.D. Thesis, Stanford University, 1988

[23] V. Joly, F. Coquel, C. Marmignon, W. Aretz, S. Metz, H. Wilhelmi, Numerical modelling of heat transfer and relaxation in nonequilibrium air at hypersonic speeds, La Recherche Aérospatiale 3 (1994) 219-234

[24] D.L. Baulch, D.D. Drysdale, D.G. Home, Evaluated kinetic data for high temparature reactions, vol. 2, Homogeneous Gas Phase Reaction of the $\mathrm{H}_{2}-\mathrm{N}_{2}-\mathrm{O}_{2}$ System, CRC Press, Butterworths, London, 1973

[25] P.H. Rose, W.I. Stark, Stagnation point heat transfer measurements in dissociated air, J. Aero. Sciences 25 (1958)

[26] P.H. Rose, J.O. Stankevics, Stagnation-point heat transfer measurements in partially ionized Air, AIAA J. 1 (1963) 2752-2763

[27] J.A. Fay, F.R. Riddell, Theory of stagnation point heat transfer in dissociated air, J. Aero. Sciences 25 (1958) 73-85

[28] E. Josyula, S.J. Shang, Computation of nonequilibrium hypersonic flowfields around hemisphere cylinders, J. Therm. Heat Trans. 7 (1993) 